\title{
Biology of gait control
}

\section{Vitamin D involvement}

O. Beauchet, $\mathrm{MD}, \mathrm{PhD}$

C. Annweiler, MD, MS

J. Verghese, MD

B. Fantino, $\mathrm{MD}, \mathrm{PhD}$

F.R. Herrmann, MD, $\mathrm{MPH}$

G. Allali, MD, PhD

Address correspondence and reprint requests to Dr. Olivier Beauchet, Department of Internal Medicine and Geriatrics, Angers

University Hospitals, 49933

Angers cedex 9, France

olbeauchet@chu-angers.fr

\section{ABSTRACT}

Background: Adverse neuromuscular events have been described in case of low serum 25hydroxyvitamin $\mathrm{D}(25 \mathrm{OHD})$ concentrations, suggesting that vitamin $\mathrm{D}$ may be involved in gait stability. The objective of this cross-sectional study was to examine the association between stride-to-stride variability of stride time (STV) and serum 250HD concentration in adults aged 65 years and older.

Methods: STV and 25OHD concentration were assessed in 411 community-dwelling older adults (mean age $70.4 \pm 1.8$ years, $57.9 \%$ women). The following established $250 \mathrm{HD}$ thresholds were used: severe $250 \mathrm{HD}$ insufficiency $<10 \mathrm{ng} / \mathrm{mL}$, moderate $10-30 \mathrm{ng} / \mathrm{mL}$, and normal $>30 \mathrm{ng} / \mathrm{mL}$. Age, number of drugs used per day, use of psychoactive drugs, depressive symptoms, cognitive decline, history of falls, distance visual acuity, lower limb proprioception, center of mass (CoM) motion, and walking speed were considered as potential confounders.

Results: A total of 16.6\% ( $n=68$ ) of subjects had severe $250 \mathrm{HD}$ insufficiency, $70.3 \%(n=289)$ moderate insufficiency, and $13.1 \%(n=54)$ normal concentrations. In the full adjusted and the stepwise backward linear regression models, high STV (worse performance) was associated with severe 250HD insufficiency ( $p=0.028$ and $p=0.044$, respectively), high CoM motion ( $p=$ 0.031 and $p=0.014$, respectively), and low lower limb proprioception score $(p=0.017$ and $p=$ 0.008 , respectively). The stepwise backward regression model also showed that high STV was associated with female gender $(p=0.041)$.

Conclusions: Low serum 250HD concentrations were associated with high STV reflecting a disturbed gait control. This association could be explained by a possible action of vitamin D on different components involved in gait control. Neurology ${ }^{\circledR} 2011 ; 76: 1617-1622$

\section{GLOSSARY}

$\mathbf{2 5 0 H D}=$ 25-hydroxyvitamin D; $\mathbf{A P}=$ anterior-posterior; $\mathbf{C o M}=$ center of mass; $\mathbf{C V}=$ coefficient of variation; $\mathbf{G D S}=$ Geriatric Depression Scale; $\mathbf{M L}=$ medial-lateral; MMSE = Mini-Mental State Examination; MVC = maximal isometric voluntary contraction; PD = Parkinson disease; STV = stride-to-stride variability of stride time.

While walking at normal self-selected speed, a subject may reproduce comparable limbcoordinated movements from stride to stride. ${ }^{1,2}$ Stride-to-stride variability of stride time (STV) is a measure of this reliability of limb movements. ${ }^{1,3}$ Because gait is an automated regular rhythmic motor behavior, STV is typically low in healthy subjects. ${ }^{1-3}$ The past decade has highlighted that STV is a dependable marker of gait control, and may be used as a clinical index of gait stability. ${ }^{1-4}$ An increase in STV has been associated with various adverse outcomes such as falls, lower limb peripheral neuropathy, Parkinson disease (PD), and dementia., ${ }^{3,5-10}$

Low levels of serum 25-hydroxyvitamin D (25OHD) is common in elderly populations, and has also been linked to risk of various adverse outcomes such as falls or low physical performance. ${ }^{11-13}$ Recently, it has been shown in a cohort of community-dwelling older women 
that low serum 25OHD concentrations were associated with low walking speed measured at usual pace as well as at fast pace. ${ }^{11}$ This last result underlined that hypovitaminosis-D contributes to the mechanism of fall by its action on neuromuscular systems involved in the control of gait stability. ${ }^{11-14}$

Based on these previous findings, we hypothesized that low serum $25 \mathrm{OHD}$ concentrations could be associated with a disturbed control of gait stability illustrated by an increase in STV. The aim of this study was to examine the association between STV and serum $25 \mathrm{OHD}$ concentrations in a populationbased sample of community-dwelling adults aged 65 years and older.

METHODS Subjects. Between June and December 2009, among the 661 community-dwelling adults aged 65 years and older who presented for a free medical examination for the French health insurance purposes at the health examination center of Lyon (France), 411 (62.2\%) were included in this study. Exclusion criteria for this cross-sectional study included age below 65 years, institutionalization, inability to understand and speak French, acute medical illness during the past month, history of dementia, and inability to walk 6 meters unassisted.

Clinical assessment. Participants underwent a full examination. The number of drugs used per day and the use of psychoactive drugs including benzodiazepines, antidepressants, or neuroleptics were recorded. They were interviewed using a standardized questionnaire, gathering information on the history of falls over the past year. A fall was defined as an event resulting in a person coming to rest unintentionally on the ground or at other lower level, not as the result of a major intrinsic event or an overwhelming hazard. Depression was evaluated by the 4-item short Geriatric Depression Scale (GDS) score. ${ }^{15}$ A score $\geq 1$ indicated symptoms of depression. Cognitive decline was considered when the subject had an abnormal Clock Drawing Test and an abnormal episodic memory test. ${ }^{16}$ Episodic memory test was built from the 6 memory items of Folstein's Mini-Mental State Examination (MMSE). ${ }^{17}$ It was calculated as (immediate recall of the 3 recall items + delayed recall of the 3 words). The score ranged from 0 to 6,6 indicating a normal score. A score $<6$ was considered as abnormal. The maximal isometric voluntary contraction (MVC) strength of dominant hand was measured with computerized hydraulic dynamometers (Martin Vigorimeter, Medizin Tecnik, Tutlingen, Germany). The test was performed 3 times consecutively. The mean value calculated from the 3 trials was used in the present data analysis. The standing postural sway on a firm surface was measured using a force platform $\left(101 \times 101 \mathrm{~cm}\right.$; BioRescue, Dune ${ }^{\circledR}$, France $)$. This instrument used vertical forces transducers to determine instantaneous fluctuations in the center of mass (CoM). The participant was asked to maintain barefoot standing position with eyes opened and each foot positioned against a foot frame on a platform plate that maintained the distance between the medial sides of the heel at $8.4 \mathrm{~cm}$ with an external rotation angle $9^{\circ}$. Subjects were instructed to look straight ahead, with arms kept by the side of the body, for 30 seconds. From the balance system software, the total CoM movement in the anterior-posterior (AP) and mediallateral (ML) directions were recorded in millimeters. Lower limb proprioception was evaluated with a $64-\mathrm{Hz}$ graduated tuning fork placed on the tibial tuberosity, and graded from 0 to 8 ( 8 considered as a normal value). The mean value obtained for the left and right sides was used in the present data analysis. Binocular visual acuity was measured at $5 \mathrm{~m}$ with a Snellen letter test chart. ${ }^{18}$ Vision was assessed with corrective lenses on if usually used by the subject. The walking speed $(\mathrm{cm} / \mathrm{s})$ was calculated from the performance of the Timed Up \& Go test. ${ }^{19}$ This test measures in seconds the time it takes a subject to rise from a chair, walk a distance of 3 meters, turn, walk back to the chair, and sit down.

Gait recordings. STV was measured at steady state walking using SMTEC $^{\circledR}$ system $\left(\right.$ SMTEC $^{\circledR}$, Sport \& Medical Technologies SA, Nyon, Switzerland) in a 4-meter corridor. The SMTEC ${ }^{\circledR}$ system consists of 2 footswitches providing a continuous measurement of temporal step parameters. ${ }^{20}$ This reliable system is a pair of innersoles fitted inside the subject's shoes. Each innersole contains 2 independent footswitches placed at the heel and the toe, which are linked to a portable data logger worn at the waist. Subjects walked one trial at their usual self-selected walking speed in a quiet, well-lit room wearing their own footwear according to European guidelines for spatio-temporal gait analysis in older adults. ${ }^{21}$ Coefficient of variation (CV) (CV = $[\mathrm{SD} /$ mean $] \times 100)$ of stride time was used to explore the outcome measure of STV.

Serum 250HD measures. Fasting early morning venous blood was collected from resting subjects for the measurement of serum 25OHD. All 25OHD measurements were performed in the same laboratory at Lyon, France. Serum concentrations of 25OHD were measured by radioimmunoassay (Incstar Corp., Stillwater, MN). With this method, there is no interference of lipids, which is often observed in other nonchromatographic assays of 25OHD. ${ }^{11}$ The intra-assay and interassay precisions for $25 \mathrm{OHD}$ in our laboratory were respectively $5.2 \%$ and $11.3 \%$ (range in normal adults aged $20-60$ years, $30-125 \mathrm{ng} / \mathrm{mL}$ ).

Statistics. The subjects' baseline characteristics were summarized using means and standard deviations or frequencies and percentages, as appropriate. Normality of data distribution was checked using skewness-kurtosis test. As the number of observations was $>40$ for each group, no transformations were applied to the variables of interest. For the current analysis, the following previously established cutoff points were used to categorize subjects into 3 groups: severe $25 \mathrm{OHD}$ insufficiency $<10 \mathrm{ng} / \mathrm{mL}$, moderate 25OHD insufficiency between 10 and $30 \mathrm{ng} / \mathrm{mL}$, and normal concentrations $>30 \mathrm{ng} / \mathrm{mL}{ }^{11}$ Comparisons among groups were performed using one-way analysis of variance, the Kruskal-Wallis test, or $\chi^{2}$, as appropriate. Multiple linear regression analyses were performed to specify the association between STV (dependent variable) and serum 25OHD (independent variable) adjusted on subjects' baseline characteristics (age, gender, drugs, falls in the past year) and other potential confounders (cognitive impairment, depressive symptoms, CoM motion, handgrip strength, lower limb proprioception, visual acuity, and walking speed). $p$ Values less than 0.05 were considered as statistically significant. All statistics were performed using the Stata Statistical Software (version 11.1; Stat Corp, College Station, TX).

Standard protocol approvals, registrations, and patient consents. Participants in the study were included after having given their written informed consent for research. The 
Table 1 Clinical characteristics of study subjects according to serum $250 \mathrm{HD}$ concentrations $(\mathrm{n}=411)$

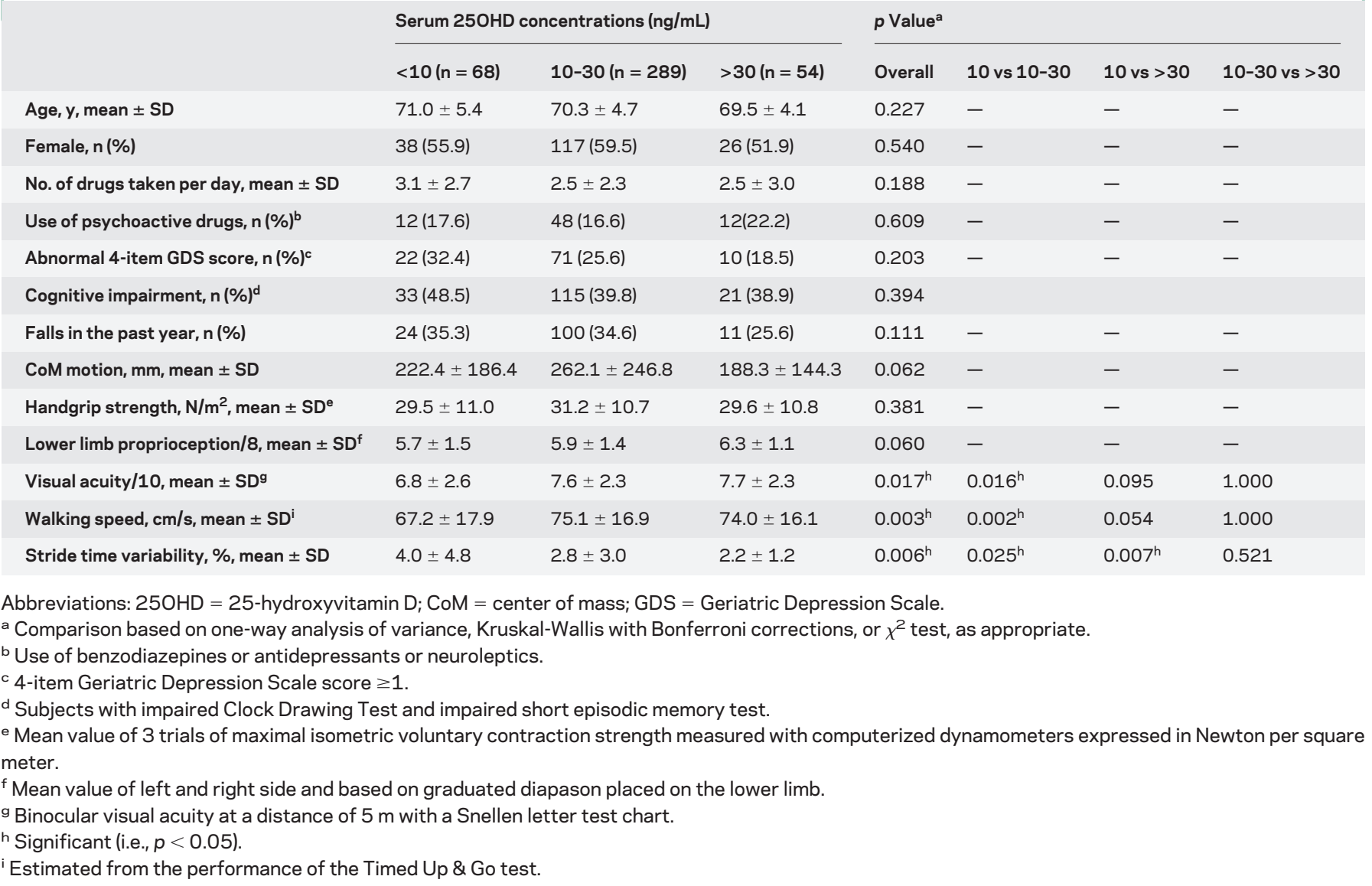

study was conducted in accordance with the ethical standards set forth in the Helsinki Declaration (1983). The entire study protocol was approved by the local Ethical Committee of Lyon (France) and the study is in compliance with the STROBE statement guidelines.

RESULTS Based on the established cutoff points for vitamin D status, ${ }^{11} 16.6 \%(\mathrm{n}=68)$ of our subjects had severe 25OHD insufficiency, $70.3 \%(\mathrm{n}=289)$ moderate 25OHD insufficiency, and 13.1\% ( $\mathrm{n}=$ 54) normal serum $25 \mathrm{OHD}$ concentrations. Subjects with severe 25OHD insufficiency had lower binocular visual acuity $(p=0.016)$ and walking speed $(p=$ $0.002)$ compared to those with moderate $25 \mathrm{OHD}$ insufficiency (table 1). STV was higher in subjects with severe 25OHD insufficiency compared to those with moderate 25OHD insufficiency $(p=0.025$ ) and those with normal serum concentrations ( $p=$ 0.007). There was no significant difference among groups in regards to the other clinical characteristics (table 1).

As shown in table 2, the full adjusted and the stepwise backward linear regression models highlighted that high STV was associated with severe 25OHD insufficiency ( $p=0.028$ and $p=0.044$, respectively), high CoM motion ( $p=0.031$ and $p=$ 0.014 , respectively), and low lower limb propriocep- tion score ( $p=0.017$ and $p=0.008$, respectively). Finally, the stepwise backward regression model showed that high STV was associated with female gender $(p=0.041)$. There was no association with STV for the other covariates in the model.

DISCUSSION Our results show that STV was higher in subjects with severe 25OHD insufficiency compared to those with normal serum $25 \mathrm{OHD}$ concentration. We highlighted that the association between increased STV and severe 25OHD insufficiency holds true even when the effects of potential confounders were taken into account. Our findings suggest a relationship between gait control assessed by STV and a specific biological marker, such as serum 25OHD concentration. This association may be explained by combined adverse effects of severe 25OHD insufficiency on the different subsystems involved in gait control including the muscular component, the neurofeedback component, and the CNS component.

Epidemiologic studies have showed mixed results for the muscular action of 25OHD. While some studies found an association between muscle weakness and serum 25OHD insufficiency, others failed to show any association. ${ }^{22-28}$ This last point is in con- 
Table 2 Multiple linear regression models showing the association between stride-to-stride variability of stride time (dependent variable) and serum 250HD concentrations (independent variable) adjusted on clinical characteristics $(n=411)$

\begin{tabular}{|c|c|c|c|c|c|c|}
\hline & $\boldsymbol{\beta}$ & $95 \% \mathrm{Cl}$ & $p$ Value & $\beta$ & $95 \% \mathrm{Cl}$ & $p$ Value \\
\hline$>30$ & 0.000 ref $^{a}$ & & & - & - & - \\
\hline $10-30$ & 0.005 & -0.004 to 0.014 & 0.267 & - & - & - \\
\hline Female & 0.007 & -0.018 to 0.004 & 0.185 & $0.007^{b}$ & 0.001 to $0.013^{b}$ & $0.041^{b}$ \\
\hline Number of drugs taken per day & 0.001 & -0.001 to 0.002 & 0.386 & - & - & - \\
\hline Use of psychoactive drugs ${ }^{c}$ & 0.005 & -0.005 to 0.015 & 0.329 & - & - & - \\
\hline Abnormal 4-item GDS score ${ }^{d}$ & 0.001 & -0.006 to 0.009 & 0.727 & - & - & - \\
\hline Handgrip strength ${ }^{f}$ & 0.00004 & -0.001 to 0.000 & 0.868 & - & - & - \\
\hline Lower limb proprioceptiong & $-0.003^{b}$ & -0.005 to $-0.001^{b}$ & $0.017^{b}$ & $-0.003^{b}$ & -0.005 to $-0.001^{b}$ & $0.008^{b}$ \\
\hline Visual acuity ${ }^{h}$ & 0.001 & -0.001 to 0.002 & 0.289 & - & - & - \\
\hline Walking speedi & -0.012 & -0.033 to 0.008 & 0.231 & -0.015 & -0.033 to 0.003 & 0.107 \\
\hline
\end{tabular}

Abbreviations: $250 \mathrm{HD}=25$-hydroxyvitamin $\mathrm{D} ; \beta=$ coefficient of regression $\beta$ corresponding to an increase or a decrease of stride time variability; $\mathrm{Cl}=$ confidence interval; $\mathrm{CoM}=$ center of mass; $\mathrm{GDS}=$ Geriatric Depression Scale.

a Normal 250HD serum concentration used as reference level.

${ }^{\mathrm{b}}$ Coefficient of regression $(\beta)$ significant.

c Use of benzodiazepines or antidepressants or neuroleptics.

d 4-item Geriatric Depression Scale score $\geq 1$.

e Subjects with impaired Clock Drawing Test and impaired short episodic memory test.

${ }^{f}$ Mean value of 3 trials of maximal isometric voluntary contraction strength measured with computerized dynamometers expressed in Newton per square meter.

9 Mean value of left and right side and based on graduated diapason placed on the lower limb.

${ }^{h}$ Binocular visual acuity at a distance of $5 \mathrm{~m}$ with a Snellen letter test chart.

'Estimated from the performance of the Timed Up \& Go test.

cordance with the absence of between-group difference of handgrip strength shown in our study. The main explanation for these mixed results could be that not muscular strength but muscular power is related to vitamin D metabolism, as suggested by Annweiler et al. ${ }^{11,12,28}$ Muscular power is defined by muscular contraction speed. A clinical consequence of the decrease in muscular power is a decrease in walking speed. ${ }^{12,28}$ Precisely, walking speed could be considered as a surrogate measure of lower limb's muscular power in this studied sample of healthy older adults. ${ }^{11,29}$ This is one reason why walking speed is different across the 3 groups of subjects, the lowest walking speed being found among subjects with the lowest $25 \mathrm{OHD}$ concentrations. Nevertheless, walking speed should not be linked only to muscular power but also depends on the nervous system. It is therefore unlikely that the influence of vitamin $\mathrm{D}$ on the muscular component is enough to explain the involvement of vitamin $\mathrm{D}$ in gait control illustrated by STV, insomuch that STV is not significantly associ- ated with walking speed in our regression models ( $p=$ 0.231 and $p=0.107$ ).

Control of gait stability also depends on a neurofeedback component, especially lower limb proprioception, postural sway, and visual acuity. It is interesting to note that serum $25 \mathrm{OHD}$ concentrations varied according to each of these neurofeedback subsystems. First, the lower limb proprioception was abnormal in the 3 groups of subjects and was the lowest in subjects with severe 25OHD insufficiency. There was a near significant difference across the 3 patient groups $(p=0.060)$. This result is supported by disturbed nerve conduction in case of severe 25OHD insufficiency in humans. ${ }^{24}$ Second, the trend for the standing postural sways across the 3 vitamin $\mathrm{D}$ groups $(p=0.062)$ and the association between this parameter and STV ( $p=0.031$ and $p=0.014)$ highlights the importance of afferent feedback for the control of gait stability. These results are in concordance with previous reported data that showed a correlation between body sways and 
serum $25 \mathrm{OHD}$ concentration in humans..$^{30}$ Finally, we observed an interesting between-group difference in visual acuity with the best visual acuity among the group with the highest serum 25OHD concentrations. This result corroborates with the observation that calcitriol may protect against age-related macular degeneration in humans. ${ }^{31}$ The influence of vitamin $\mathrm{D}$ on the neurofeedback components could therefore explain in part the involvement of vitamin $\mathrm{D}$ in postural and gait control. Nevertheless, we found an association between STV and serum vitamin D status in spite of the adjustment on lower limb proprioception, standing postural sways, and visual acuity. This meant that the vitamin D-related neurofeedback component is also not enough to explain the control of gait.

At the brain level, by its action on different neurotransmitters and several growth factors, low serum 25OHD has been associated with low global cognitive function ${ }^{30,32-35}$ that was likely explained by hypovitaminosis $\mathrm{D}$-induced executive dysfunction and processing speed impairment. ${ }^{14,32}$ Previous studies have highlighted the importance of the executive function for gait control ${ }^{36,37}$ and have suggested that STV could be a suitable parameter to assess the executive component of gait control. ${ }^{10}$ STV is also indicative of specific basal ganglia involvement in the gait control, as suggested by the increased STV seen in patients with PD. ${ }^{37}$ A close link between hypovitaminosis D-related executive dysfunction and impaired gait control illustrated by an increased STV is therefore likely. In the present study, cognitive impairment levels do not differ among the 3 groups of vitamin $\mathrm{D}$, which could be explained by the fact that we did not include patients with dementia and that cognitive assessment was limited to a brief assessment of episodic memory and executive function. This limitation could also explain why our cognitive measure was not associated with STV $(p=0.924)$.

Our study has some limitations. First, the study cohort was restricted to healthy volunteers who could be not representative of community-dwelling older adults and probably more motivated with greater interest in personal health issues. Secondly, the crosssectional design did not allow any causal inferences. For instance, high STV may lead to a loss of independence with low dietary intakes of vitamin $\mathrm{D}$ and a lack of sunlight exposure, which in turn leads to low vitamin D status. A scenario of reverse causation remains possible. Hence, longitudinal studies are needed to follow up on our initial promising results to address issues of causality. Third, although we controlled for many characteristics that are likely to modify the association between STV and serum 25OHD concentration, residual confounders—such as the listing of arthritic manifestations - may still be present. Fourth, limitations of this study include the use of a $2 \times 3$-meter walkway test rather than a 6-meter walkway test for the calculation of gait speed. Finally, although we assessed the main components involved in gait control, residual components of gait control could be missed.

Low serum 25OHD concentrations were associated with high STV, which is a sensitive measure of gait control. This association could be explained by a possible action of vitamin $\mathrm{D}$ on different components involved in gait control. High STV could therefore represent an interesting marker of serum 25OHD insufficiency. Subjects with high STV should be checked for vitamin D insufficiency and substituted if necessary. Our results add a new orientation of research into furthering the understanding of how vitamin $\mathrm{D}$ affects motor coordination and locomotion.

\section{AUTHOR CONTRIBUTIONS}

Statistical analysis was conducted by Dr. F.R. Herrmann. Dr. Beauchet has full access to all of the data in the study, takes responsibility for the data, the analyses, and interpretation, and has the right to publish any and all data, separate and apart from the attitudes of the sponsor. All authors meet all of the following criteria: 1) contributing to the conception and design, or analyzing and interpreting data; 2) drafting the article or revising it critically for important intellectual content; and 3) approving the final version to be published.

\section{ACKNOWLEDGMENT}

The authors thank the participants for their cooperation.

\section{DISCLOSURE}

Prof. Beauchet serves on the editorial board of Annales de Gérontologie and serves as a consultant for the Korian Group. Dr. Annweiler serves as a consultant for Ipsen. Dr. Verghese has served on the speakers' bureau for Pfizer Inc and receives research support from the NIH/NIA, the American Federation of Aging Research, and the Donald W Reynolds Foundation. Dr. Fantino reports no disclosures. Dr. Herrmann serves on editorial advisory boards for Current Gerontology and Geriatrics Research, European Geriatrics Medicine, and Annales de Gérontologie. Dr. Allali has received research support from the Swiss National Science Foundation.

\section{Received September 1, 2010. Accepted in final form December 17, 2010.}

\section{REFERENCES}

1. Hausdorff JM. Gait dynamics, fractals and falls: finding meaning in the stride-to-stride fluctuations of human walking. Hum Mov Sci 2007;26:555-589.

2. Beauchet O, Allali G, Annweiler C, et al. Gait variability among healthy adults: low and high stride-to-stride variability are both a reflection of gait stability. Gerontology 2009;55:702-706.

3. Gabell A, Nayak US. The effect of age on variability in gait. J Gerontol 1984;39:662-666.

4. Beauchet O, Allali G, Berrut G, Dubost V. Is low lowerlimb kinematic variability always an index of stability? Gait Posture 2007;26:327-328.

5. Hausdorff JM, Rios DA, Edelberg HK. Gait variability and fall risk in community-living older adults: a 1-year 
prospective study. Arch Phys Med Rehabil 2001;82: 1050-1056

6. Maki BE. Gait changes in older adults: predictors of falls or indicators of fear. J Am Geriatr Soc 1997;45:313-320.

7. Snijders AH, van de Warrenburg BP, Giladi N, Bloem BR. Neurological gait disorders in elderly people: clinical approach and classification. Lancet Neurol 2007;6:63-74.

8. Hausdorff JM, Cudkowicz ME, Firtion R, Wei JY, Goldberger AL. Gait variability and basal ganglia disorders: stride-to-stride variations of gait cycle timing in Parkinson's disease and Huntington's disease. Mov Disord 1998; 13:428-437.

9. Richardson JK, Thies SB, DeMott TK, Ashton-Miller JA. A comparison of gait characteristics between older women with and without peripheral neuropathy in standard and challenging environments. J Am Geriatr Soc 2004;52: 1532-1537.

10. Allali G, Dubois B, Assal F, et al. Frontotemporal dementia: pathology of gait? Mov Disord 2010;25:723-729.

11. Annweiler C, Schott AM, Montero-Odasso M, et al. Cross-sectional association between serum vitamin $\mathrm{D}$ concentration and walking speed measured at usual and fast pace among older women: The EPIDOS study. J Bone Miner Res 2010;25:1858-1866.

12. Annweiler C, Schott AM, Berrut G, Fantino B, Beauchet O. Vitamin D-related changes in physical performance: a systematic review. J Nutr Health Aging 2009;13:893898.

13. Annweiler C, Montero-Odasso M, Schott AM, Berrut G, Fantino B, Beauchet O. Fall prevention and vitamin D in the elderly: an overview of the key role of the non-bone effects. J Neuroeng Rehabil 2010;7:50.

14. Annweiler C, Allali G, Allain P, et al. Vitamin D and cognitive performance in adults: a systematic review. Eur J Neurol 2009;16:1083-1089.

15. Shah A, Herbert R, Lewis S, Mahendran R, Platt J, Bhattacharyya B. Screening for depression among acutely ill geriatric inpatients with a short Geriatric Depression Scale. Age Ageing 1997;26:217-221.

16. Cahn DA, Salmon DP, Monsch AU, et al. Screening for dementia of the Alzheimer type in the community: the utility of the Clock Drawing Test. Arch Clin Neuropsychol 1996;11:529-539.

17. Folstein MF, Folstein SE, McHugh PR. "Mini-mental state": a practical method for grading the cognitive state of patients for the clinician. J Psychiatr Res 1975;12:189-198.

18. Lord SR, Ward JA, Williams P, et al. Physiological factors associated with falls in older community-dwelling women. J Am Geriatr Soc 1994;42:1110-1117.

19. Podsiadlo D, Richardson S. The timed "Up \& Go": a test of basic functional mobility for frail elderly persons. J Am Geriatr Soc 1991;39:142-148.

20. Beauchet O, Herrmann FR, Grandjean R, Dubost V, Allali G. Concurrent validity of SMTEC footswitches system for the measurement of temporal gait parameters. Gait Posture 2008;27:156-159.

21. Kressig RW, Beauchet O, European GAITRite Network Group. Guidelines for clinical applications of spatiotemporal gait analysis in older adults. Aging Clin Exp Res 2006;18:174-176.

22. Costa EM, Blau HM, Feldman D. 1,25-dihydroxyvitamin D3 receptors and hormonal responses in cloned human skeletal muscle cells. Endocrinology 1986;119:22142220.

23. Norman AW, Nemere I, Zhou LX, et al. 1,25(OH)2vitamin D3, a steroid hormone that produces biologic effects via both genomic and nongenomic pathways. J Steroid Biochem Mol Biol 1992;41:231-240.

24. Skaria J, Katiyar BC, Srivastava TP, Dube B. Myopathy and neuropathy associated with osteomalacia. Acta Neurol Scand 1975;51:37-58.

25. Bischoff-Ferrari HA, Dietrich T, Orav EJ, et al. Higher 25-hydroxyvitamin $\mathrm{D}$ concentrations are associated with better lower-extremity function in both active and inactive persons aged $>$ or $=60 \mathrm{y}$. Am J Clin Nutr 2004;80:752758.

26. Chou FF, Chee EC, Lee CH, Sheen-Chen SM. Muscle force, motor nerve conduction velocity and compound muscle action potentials after parathyroidectomy for secondary hyperparathyroidism. Acta Neurol Scand 2002; 106:218-221.

27. Wicherts IS, van Schoor NM, Boeke AJ, et al. Vitamin D status predicts physical performance and its decline in older persons. J Clin Endocrinol Metab 2007;92:20582065.

28. Annweiler $\mathrm{C}$, Beauchet $\mathrm{O}$, Berrut $\mathrm{G}$, et al. Is there an association between serum 25-hydroxyvitamin $\mathrm{D}$ concentration and muscle strength among older women? Results from baseline assessment of the EPIDOS study. J Nutr Health Aging 2009;13:90-95.

29. Bonnefoy M, Jauffret M, Jusot JF. Muscle power of lower extremities in relation to functional ability and nutritional status in very elderly people. J Nutr Health Aging 2007;11: 223-228.

30. Dhesi JK, Bearne LM, Moniz C, et al. Neuromuscular and psychomotor function in elderly subjects who fall and the relationship with vitamin D status. J Bone Miner Research 2002;17:891-899.

31. Parekh N, Chappell RJ, Millen AE, Albert DM, Mares JA. Association between vitamin $\mathrm{D}$ and age-related macular degeneration in the Third National Health and Nutrition Examination Survey, 1988 through 1994. Arch Ophthalmol 2007;125:661-669.

32. Annweiler C, Schott AM, Berrut G, et al. Vitamin D and ageing: neurological issues. Neuropsychobiology 2010;62: $139-150$.

33. Annweiler C, Schott AM, Allali G, et al. Association of vitamin $\mathrm{D}$ deficiency with cognitive impairment in older women: cross-sectional study. Neurology 2010;74:27-32.

34. Annweiler C, Le Gall D, Fantino B, Beauchet O, Tucker KL, Buell JS. 25-hydroxyvitamin D, dementia, and cerebrovascular pathology in elders receiving home services. Neurology 2010;75:95.

35. Buell JS, Dawson-Hughes B, Scott TM, et al. 25Hydroxyvitamin D, dementia, and cerebrovascular pathology in elders receiving home services. Neurology 2010;74: $18-26$.

36. Beauchet O, Allali G, Berrut G, Hommet C, Dubost V, Assal F. Gait analysis in demented subjects: Interests and perspectives. Neuropsychiatr Dis Treat 2008;4:155-160.

37. Yogev-Seligmann G, Hausdorff JM, Giladi N. The role of executive function and attention in gait. Mov Disord 2008;23:329-342. 